\title{
Decoupled control of posture and trajectory of the hybrid wheel-legged robot Hylos
}

\author{
Ch. Grand, F. BenAmar, F. Plumet and Ph. Bidaud \\ Laboratoire de Robotique de Paris - CNRS FRE2507 \\ Université de Paris 6, Paris, France \\ Email: \{grand, amar, plumet, bidaud $\} @$ robot.jussieu.fr
}

\begin{abstract}
This paper addresses the control of a hybrid wheellegged system evolving on rough terrain. First, the posture and trajectory parameters are introduced. Then, a decoupled posture and trajectory control algorithm based on the velocity model of the robot is proposed. Last, the performance and feasibility of the control algorithm are evaluated through simulations and experiments with the Hylos robot.
\end{abstract}

\section{INTRODUCTION}

Autonomous exploration missions require mobile robots that can carry out high performance locomotion tasks while insuring the system integrity. For applications such as planetary or volcanic exploration or various missions in hazardous areas or construction sites, the locomotion performances in terms of power consumption, autonomy and reliability are of first importance. Vehicle motion on uneven surfaces involves complex wheel-ground interactions that are related to the geometrical and physical soil properties: roughness, rocks distribution, soil compaction, friction characteristics, etc... Therefore, enhancing the locomotion performances in such environment requires the design of innovative locomotion systems and the research of original control schemes.

Available locomotion systems can roughly be divided into wheeled, tracked and legged systems. For wheeled robots evolving on natural rough terrain, the main research activity concerns the design of innovative steering (Nomad[1]) and suspension systems. The use of passive suspension systems for high terrain adaptability can be illustrated by the Rocky rovers developed at the JPL[2] or Shrimp developed at the EPFL[3]. They can be seen as wheeled systems with passive mobilities allowing the vehicle to address more challenging terrain including ground discontinuities that are higher than the wheel radius. The main advantage of wheeled locomotion systems is their performances in terms of power consumption, velocity and available payload. Legged systems have been considered for a long time as a possible way to increase the field of accessible terrains for autonomous vehicles [4], [5]. Research activities in this field concern the control of complex kinematics structure by considering gait schemes and stability margin. The main relevance of walking machines is their abilities to adapt their posture on uneven terrain and to cross over high terrain discontinuities.

Among these classical categories of locomotion mechanisms, a new way exists for hybrid vehicles, which consists in a combination of the first solutions. They can be seen as articulated vehicle with active internal mobilities and can be illustrated by the system WAAV[6] (Wheeled Actively Articulated Vehicle) or the Marsokhod[7], [8] robot. Typical hybrid wheeled, tracked and legged vehicles can be illustrated by the Roller-Walker[9], the Workpartner[10] or more recently the robot Azimut[11], which are able to combine different locomotion modes. Another approach is proposed in [12] with the compliant-legged hexapod Rhex. In this paper, we consider the hybrid wheel-legged vehicle Hylos[13] developed at LRP and we will focus on the control of this robot.

The control of such locomotion systems is not a trivial problem since we must find algorithms and strategies for the control of a redundantly actuated system ${ }^{1}$ exhibiting complex interactions with the environment. The kineto-static analyses of such complex vehicle have already been addressed by previous authors[6], [14]. Solutions for the WAAV's specific kinematics are presented and studied in simulation. This mathematical analysis leads to a model based control [15] that considers the problem of contact forces distribution in the case of the GOFOR mini-rover, which have four internal mobility degrees. However, this work considers only planar vehicle motion and was not experimentally validated. More recently, the research on control of articulated suspension vehicle was also considered [16]. The authors proposed a method for stabilitybased articulated suspension control that is experimentally demonstrated on the SRR robot of the JPL. They address the tipover stability in the case of the SRR robot that possesses two internal mobilities. By considering also the motion of a three degrees of freedom arm manipulator mounted on the platform they improve the vehicle tipover stability.

In this paper, we propose a method for the control of both the trajectory and the posture of the wheel-legged robot Hylos (see Fig. 10). This method is based on the inverse velocity model of the vehicle. In Section II, we introduce the kinematic parameters and develop the general velocity model of a wheellegged vehicle. Then, the specific inverse velocity model of Hylos platform is proposed. In Section III, we propose a kinematically decoupled control algorithm of both the vehicle trajectory and posture. In the last section, the performance of this control algorithm is analyzed through simulations and

\footnotetext{
${ }^{1}$ the number of actuated degrees of freedom is greater than the dimension of the system workspace
} 
experiments of the Hylos robot evolving on an irregular and sloping ground.

\section{SYSTEM MODELING}

This section deals with the development of direct and inverse velocity model of the wheel-legged robot Hylos. It is a lightweight mini-robot and it has 16 actuated degrees of freedom with four wheel-legs, each one combining a two degrees of freedom suspension mechanism and a steering and driven wheel. The models developed in this section will be used for the posture and trajectory control of vehicle evolving on rough terrain.

The system is composed by a main body (the platform $\mathrm{S}$ ) connected to serial articulated chains ended by a cylindrical wheel (Fig. 1). Lets us define $\mathcal{R}=(\mathrm{G}, \mathbf{x}, \mathbf{y}, \mathbf{z})$ a frame attached to the platform. The platform center-of-gravity (c.o.g) is denoted $\mathrm{G}$. The orientation of the platform frame is given by three angles with respect to the fixed frame $\mathcal{R}_{0}$, which are the conventional yaw $(\theta)-\operatorname{pitch}(\psi)-\operatorname{roll}(\varphi)$ angles[17].

\section{A. Posture and trajectory parameters}

The number of posture parameters is related to the degree of mobility of the vehicle which depends on the particular design of Hylos robot. The degree of mobility is computed using the Kutzbach form of Gruebler's equation:

$$
m=\sum_{i=0}^{j} f_{i}-6(j-b+1)
$$

where $b$ is the number of bodies, $j$ is the number of joints and $f_{i}$ is number of freedom for each joint.

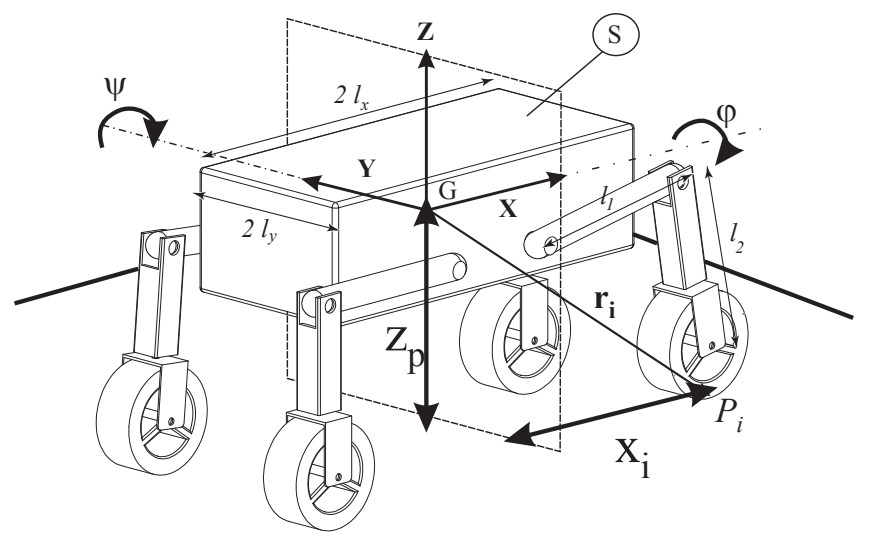

Fig. 1. Hylos posture parameters

The Hylos specific kinematics presented on Fig. 1 has a mobility degree $m=10$ with 16 actuated joints: $b=18$ (4 bodies for each leg, the platform and the ground), $j=20$ (4 joints on each leg +4 wheel-ground contacts) and $\sum f_{i}=28$ (4 rotational joints for each leg and 3 degrees of freedom joints at each wheel-ground contact considering ideal rolling constraint).
These 10 mobilities correspond in the operational velocity space to the 6 platform parameters $\mathbf{v}_{\mathbf{p}}$ and to the 4 wheelbase velocities $\dot{x}_{i}$ of each contact (Fig. 1). These parameters can be split in one part dealing with path tracking $\mathbf{v}_{\mathbf{t}}=$ $\left(v_{x}, v_{y}, \omega_{z}\right)$ and the other with the posture reconfiguration $\dot{\mathbf{p}}=\left(\omega_{x}, \omega_{y}, v_{z}, \dot{x}_{1}, \dot{x}_{2}, \dot{x}_{3}, \dot{x}_{4}\right)$. Then, the corresponding geometrical parameters for the posture and the trajectory are:

$$
\begin{aligned}
& \mathbf{p}=\left(z_{g}, \varphi, \psi, x_{1}, x_{2}, x_{3}, x_{4}\right)^{t} \\
& \mathbf{u}=\left(x_{p}, y_{p}, \theta\right)^{t}
\end{aligned}
$$

where : $\theta, \psi$ and $\varphi$ are the yaw-pitch-roll angles, $x_{i}$ the wheelbase of each wheel, $x_{p}, y_{p}$ the position of the platform center of mass, and $z_{g}$ the height of platform center relative to ground which is defined as the average of contact heights $z_{i}: z_{g}=\left(\sum_{i} z_{i}\right) / 4$.

\section{B. General velocity model}

We assume that all wheels are in contact with the ground. Lets call $\mathrm{P}_{\mathrm{i}}$ the $\mathrm{i}^{\text {th }}$ contact point and $\mathbf{n}_{\mathbf{i}}$ the normal vector to the tangent contact plane. The associated contact frame $\mathcal{R}_{i}=$ $\left(\mathrm{P}_{\mathrm{i}}, \mathbf{t}_{\mathbf{i}}, \mathbf{l}_{\mathbf{i}}, \mathbf{n}_{\mathbf{i}}\right)$ is defined such as $\mathbf{t}_{\mathbf{i}}=\frac{\boldsymbol{\sigma}_{\mathbf{i}} \times \mathbf{n}_{\mathbf{i}}}{\left\|\boldsymbol{\sigma}_{\mathbf{i}} \times \mathbf{n}_{\mathbf{i}}\right\|}\left(\boldsymbol{\sigma}_{\mathbf{i}}\right.$ is the $\mathrm{i}^{\text {th }}$ wheel axis unit vector) and $\mathbf{l}_{\mathbf{i}}=\mathbf{n}_{\mathbf{i}} \times \mathbf{t}_{\mathbf{i}}$.

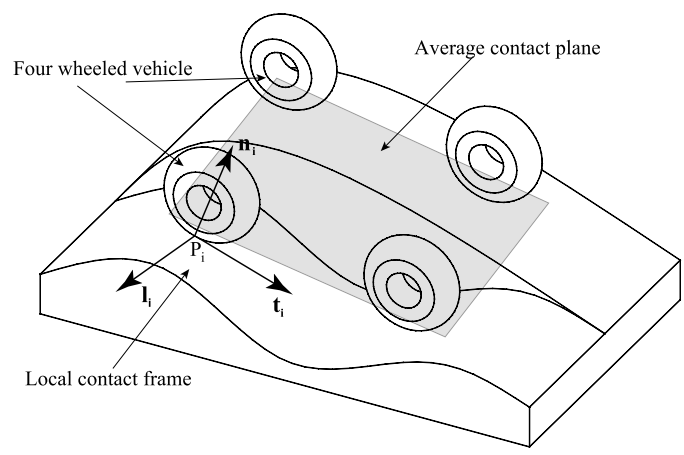

Fig. 2. Contact frame and average plane definition

The velocity of each contact point $\mathrm{P}_{\mathrm{i}}$ with respect to ground can be written as:

$$
\mathbf{v}_{\left(\mathrm{P}_{\mathrm{i}} / \mathcal{R}_{0}\right)}=\mathbf{v}+\boldsymbol{\omega} \times \mathbf{r}_{\mathbf{i}}+\mathbf{v}_{\left(\mathrm{P}_{\mathrm{i}} / \mathcal{R}\right)}
$$

where $(\mathbf{v}, \boldsymbol{\omega})^{t}=\mathbf{v}_{\mathbf{p}}$ is the components vector expressing the twist of the platform and $\mathbf{r}_{\mathbf{i}}$ is the vector connecting the platform frame center $\mathrm{G}$ to the contact point $\mathrm{P}_{\mathrm{i}}$.

Considering the pure rolling condition at contact point $\mathrm{P}_{\mathrm{i}}$ : $\mathbf{v}_{\left(\mathrm{P}_{\mathrm{i}} / \mathcal{R}_{0}\right)}=\mathbf{0}$, the equation (3) becomes:

$$
-\left(\mathbf{v}+\boldsymbol{\omega} \times \mathbf{r}_{\mathbf{i}}\right)=\mathbf{v}_{\left(\mathrm{P}_{\mathrm{i}} / \mathcal{R}\right)}
$$

and its projection along the contact frame vectors yields, in a matrix form, to:

$$
-\left(\begin{array}{ll}
\mathbf{R}_{\mathbf{i}} & -\mathbf{R}_{\mathbf{i}} \mathbf{S}\left(\mathbf{r}_{\mathbf{i}}\right)
\end{array}\right) \mathbf{v}_{\mathbf{p}}=\mathbf{J}_{\mathbf{i}} \dot{\mathbf{q}}_{\mathbf{i}}
$$

where $\mathbf{R}_{\mathbf{i}}$ is the rotation matrix of contact frame with respect to platform frame, $\mathbf{J}_{\mathbf{i}}$ is the jacobian matrix of the $\mathrm{i}^{\text {th }}$ wheelleg chain with respect to the platform and expressed in the 
contact frame, $\dot{\mathbf{q}}_{\mathbf{i}}$ is the joint velocities vector of the wheelleg chains, and $\mathbf{S}(\mathbf{a})$ is the skew-symmetric matrix of the cross product operator:

$$
\mathbf{S}(\mathbf{a})=\left(\begin{array}{ccc}
0 & a_{3} & -a_{2} \\
-a_{3} & 0 & a_{1} \\
a_{2} & -a_{1} & 0
\end{array}\right)
$$

This equation can be written as:

$$
\mathbf{L}_{\mathbf{i}} \mathbf{v}_{\mathbf{p}}=\mathbf{J}_{\mathbf{i}} \dot{q}_{\mathbf{i}}
$$

$\mathbf{L}_{\mathbf{i}}$ is called the locomotion matrix of the $\mathrm{i}^{\text {th }}$ wheel-leg chain. Finally, for all wheel-legs, we obtain:

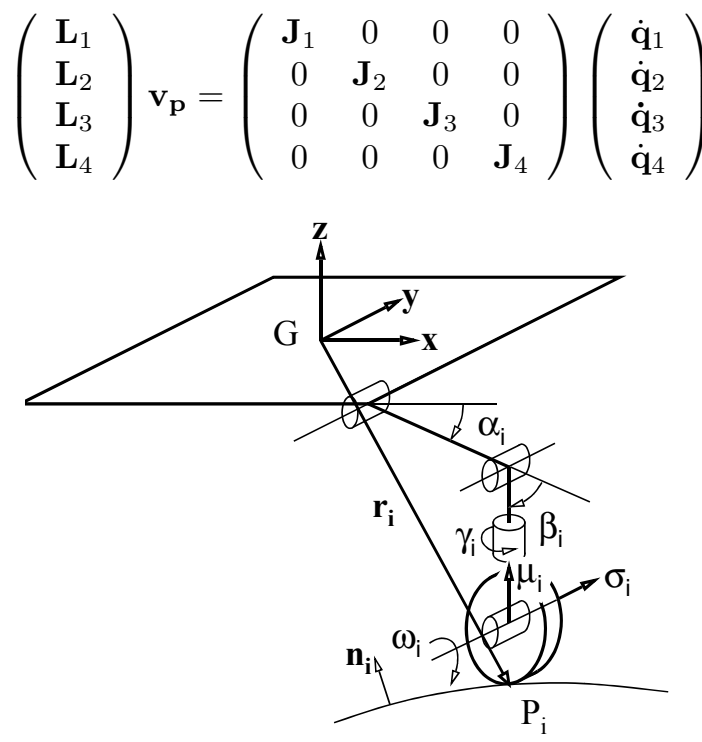

Fig. 3. Hylos - Parameters of a wheel-leg

\section{Inverse velocity model}

Let us consider the wheel-leg chain kinematics given in Fig. 3 . $\alpha_{i}, \beta_{i}$ are the leg's joint angles, $\gamma_{i}$ the steering angle and $\omega_{i}$ the wheel rate. Equation (4) expressed for the Hylos robot becomes:

$$
\begin{aligned}
-\left(\mathbf{v}+\boldsymbol{\omega} \times \mathbf{r}_{\mathbf{i}}\right)= & \dot{\alpha}_{i} \mathbf{y} \times \mathbf{a}_{\mathbf{i}}+\dot{\beta}_{i} \mathbf{y} \times \mathbf{b}_{\mathbf{i}}+\ldots \\
& \ldots \dot{\gamma}_{i} \boldsymbol{\mu}_{\mathbf{i}} \times \mathbf{c}_{\mathbf{i}}+\omega_{i} \boldsymbol{\sigma}_{\mathbf{i}} \times \mathbf{d}_{\mathbf{i}}
\end{aligned}
$$

where $\mu_{\mathbf{i}}, \sigma_{\mathbf{i}}$ are the unit vectors of the steering and wheel axes, and $\mathbf{a}_{\mathbf{i}}, \mathbf{b}_{\mathbf{i}}, \mathbf{c}_{\mathbf{i}}, \mathbf{d}_{\mathbf{i}}$ are vectors connecting the joint axis to the contact point.

Moreover, due to the particular kinematics of the steering joint, the steering axis is almost perpendicular to the contact surface. Then the cross product $\boldsymbol{\mu}_{\mathbf{i}} \times \mathbf{c}_{\mathbf{i}}=\boldsymbol{\mu}_{\mathbf{i}} \times-r_{w} \mathbf{n}_{\mathbf{i}}$ is almost null. The steering rate $\dot{\gamma}_{i}$ has no noticeable effect on the instantaneous velocity of the platform. The normal vector is assumed to be in the wheel plane, then $\sigma_{\mathbf{i}}=\mathbf{l}_{\mathbf{i}}$ and $\omega_{i} \boldsymbol{\sigma}_{\mathbf{i}} \times \mathbf{d}_{\mathbf{i}}=\omega_{i} \mathbf{l}_{\mathbf{i}} \times\left(-r_{w} \mathbf{n}_{\mathbf{i}}\right)=-r_{w} \omega_{i} \mathbf{t}_{\mathbf{i}}$. Assuming these conditions, equation (4) becomes:

$$
-\left(\mathbf{v}+\boldsymbol{\omega} \times \mathbf{r}_{\mathbf{i}}\right)=\dot{\alpha}_{i} \mathbf{y} \times \mathbf{a}_{\mathbf{i}}+\dot{\beta}_{i} \mathbf{y} \times \mathbf{b}_{\mathbf{i}}-r_{w} \omega_{i} \mathbf{t}_{\mathbf{i}}
$$

As proposed previously, this equation is projected in the $i^{\text {th }}$ contact frame:

$$
\mathbf{L}_{\mathbf{i}} \mathbf{v}_{\mathbf{p}}=\mathbf{J}_{\mathbf{i}} \dot{\boldsymbol{q}}_{\mathbf{i}}
$$

where $\mathbf{J}_{\mathbf{i}}=\left(\begin{array}{lll}\mathbf{y} \times \mathbf{a}_{\mathbf{i}} & \mathbf{y} \times \mathbf{b}_{\mathbf{i}} & -r_{w} \mathbf{t}_{\mathbf{i}}\end{array}\right)$ is the $3 \times 3$ jacobian matrix of each leg and $\dot{\mathbf{q}}_{\mathbf{i}}=\left(\dot{\alpha}_{i}, \dot{\beta}_{i}, \omega_{i}\right)^{t}$.

We remind that this equation expresses the contact condition and the non-slippage condition along the two tangential directions. The second row scalar equation expresses the inherent non-holonomic constraint of wheeled system:

$$
\boldsymbol{\sigma}_{\mathbf{i}}^{t}\left(\mathbf{v}+\boldsymbol{\omega} \times \mathbf{r}_{\mathbf{i}}+\dot{\alpha}_{i} \mathbf{y} \times \mathbf{a}_{\mathbf{i}}+\dot{\beta}_{i} \mathbf{y} \times \mathbf{b}_{\mathbf{i}}\right)=0
$$

This constraint is satisfied at each instant by computing the steering angle $\gamma_{i}$ compatible with the system motion. Thus, the scalar equation is eliminated from the matrix equation (10) by multiplying it with a reduction matrix $\mathbf{B}$ :

$$
\mathbf{B}=\left(\begin{array}{lll}
1 & 0 & 0 \\
0 & 0 & 1
\end{array}\right)
$$

By computing the $\gamma_{i}$ compatible with the system motion, we reduce the number of velocity parameters to 12 . The previous equations expressed for each four legs give only 8 scalar equations. As mentioned in previous paragraph, we introduce the wheelbase parameter changes $\dot{x}_{i}$ in order to complete the operational parameters vector. Then, the model of the $i^{\text {th }}$ wheel-legged motion can be written as:

$$
\left(\begin{array}{cc}
\mathbf{B L}_{\mathbf{i}} & \mathbf{0} \\
\mathbf{0} & 1
\end{array}\right)\left(\begin{array}{c}
\mathbf{v}_{\mathbf{p}} \\
\dot{x}_{i}
\end{array}\right)=\left(\begin{array}{c}
\mathbf{B J}_{\mathbf{i}} \\
\mathbf{j}_{\mathbf{i}}
\end{array}\right) \dot{\mathbf{q}}_{\mathbf{i}}
$$

where $\mathbf{j}_{\mathbf{i}}=\left(-l_{1} \cos \alpha_{i}-l_{2} \cos \left(\alpha_{i}+\beta_{i}\right),-l_{2} \cos \left(\alpha_{i}+\beta_{i}\right), 0\right)$ is a reduced jacobian matrix of the leg expressed in the platform frame. We then obtain:

$$
\mathbf{L}_{\mathbf{i}}^{*}\left(\begin{array}{c}
\mathbf{v}_{\mathbf{p}} \\
\dot{x}_{i}
\end{array}\right)=\mathbf{J}_{\mathbf{i}}{ }^{*} \dot{\mathbf{q}}_{\mathbf{i}}
$$

$\mathbf{J}_{\mathbf{i}}{ }^{*}$ is a $3 \times 3$ square matrix and can be inverted to give finally the wheel-leg motion by:

$$
\dot{\mathbf{q}}_{\mathbf{i}}=\left(\mathbf{J}_{\mathbf{i}}{ }^{*}\right)^{-1} \mathbf{L}_{\mathbf{i}}{ }^{*}\left(\begin{array}{c}
\mathbf{v}_{\mathbf{p}} \\
\dot{x}_{i}
\end{array}\right)
$$

The steering angle is the solution of the non-lateral slippage equation (11) which yields to the analytical form:

$$
\gamma_{i}=\arctan \left(\frac{v_{i_{y}}^{\prime}}{v_{i_{x}}^{\prime} S_{\alpha_{i}, \beta_{i}}-v_{i_{z}}^{\prime} C_{\alpha_{i}, \beta_{i}}}\right)
$$

where $v_{i_{y}}^{\prime}=v_{y}+\omega_{z} x_{i}-\omega_{x} z_{i}, v_{i_{x}}^{\prime}=v_{x}+\omega_{y} z_{i}-\omega_{z} y_{i}+\dot{x}_{i}$ and $v_{i_{z}}^{\prime}=v_{z}+\omega_{x} y_{i}-\omega_{y} x_{i}+\dot{z}_{i}$

$\left(x_{i}, y_{i}, z_{i}\right)^{t}$ are the coordinates of contact point $\mathrm{P}_{\mathrm{i}}$ in the platform frame and are expressed as:

$$
\begin{aligned}
& x_{i}= \pm l_{x}+l_{1} C_{\alpha_{i}}+l_{2} C_{\alpha_{i}, \beta_{i}} \\
& y_{i}= \pm l_{y} \\
& z_{i}=-\left(l_{1} S_{\alpha_{i}}+l_{2} S_{\alpha_{i}, \beta_{i}}+r_{w}\right)
\end{aligned}
$$

where $l_{1}$ and $l_{2}$ are the length of the leg links, $l_{x}$ and $l_{y}$ are the half length and width of the platform. 


\section{POSTURE AND TRAJECTORY CONTROL}

In this section, we describe the method used for the posture and trajectory control. We consider a proportional feedback based on the measure of actual vehicle posture $\mathbf{p}$ and position $\mathbf{u}$, which provides the reconfiguration and tracking velocity $\dot{\mathbf{p}}$ and $\mathbf{v}_{\mathbf{t}}$ as defined below. Then, the operational space velocity terms $\mathbf{v}_{\mathbf{p}}$ and $\dot{x}_{i}$ are computed as a function of these reconfiguration velocity terms by considering the velocity decoupling problem. Finally, the desired joint velocities are determined by using the inverse velocity model of the robot.

\section{A. Decoupled velocity model}

The platform angular velocities $\boldsymbol{\omega}$ are coupled functions of $(\dot{\varphi}, \dot{\psi}, \dot{\theta})^{t}$. The relation between platform rotation components $\boldsymbol{\omega}$ and rotation parameters are:

$$
\left\{\begin{array}{l}
\omega_{x}=\dot{\varphi}-\dot{\theta} \sin \psi \\
\omega_{y}=\dot{\psi} \cos \varphi+\dot{\theta} \cos \psi \sin \varphi \\
\omega_{z}=\dot{\theta} \cos \psi \cos \varphi-\dot{\psi} \sin \varphi
\end{array}\right.
$$

So, the decoupling matrix $\mathbf{D}$ is introduced in order to compute the platform velocity as a function of the derivate with respect to time of posture and trajectory parameters:

$$
\mathbf{v}_{\mathbf{p}}=\mathbf{D}\left(v_{x}, v_{y}, v_{z}, \dot{\varphi}, \dot{\psi}, \dot{\theta}\right)^{t}
$$

and

$$
\mathbf{D}=\left(\begin{array}{cccc}
\mathbf{I}_{3 \times 3} & & \mathbf{0} & \\
& 1 & 0 & -S_{\psi} \\
\mathbf{0} & 0 & C_{\varphi} & C_{\psi} S_{\varphi} \\
& 0 & -S_{\varphi} & C_{\psi} C_{\varphi}
\end{array}\right)
$$

\section{B. Posture control}

For a given optimal posture $\mathbf{p}_{\mathbf{d}}$ and a desired trajectory $\mathbf{u}_{\mathbf{d}}$, the goal of posture control is to compute the internal joint velocities $\dot{\mathbf{q}}_{\mathbf{i}}$ to apply on each motor to reach the optimal posture during the motion. Let us introduce $\dot{\mathbf{p}}=\left(\dot{\varphi}, \dot{\psi}, \dot{z}_{g}, \dot{x}_{1}, \dot{x}_{2}, \dot{x}_{3}, \dot{x}_{4}\right)^{t}$ the time-derivative of posture parameters. The posture control is achieved through a proportional feedback:

$$
\dot{\mathbf{p}}=\mathbf{K}_{\mathbf{p}} \Delta \mathbf{p}
$$

where $\Delta \mathbf{p}=\mathbf{p}_{\mathbf{d}}-\mathbf{p}$ is the posture error and $\mathbf{K}_{\mathbf{p}}$ is a $7 \times 7$ diagonal positive matrix gain.

The term $\dot{z}_{g}$ is a function of $\mathbf{v}_{\mathbf{p}}$ and, as we consider a desired posture such that the projected platform c.o.g is equivalent to the geometric center of wheel-ground contact points (see Section IV), it can be approximated:

$$
\dot{z}_{g}=v_{z}-\omega_{y} \frac{\sum_{i} x_{i}}{4}+\omega_{x} \frac{\sum_{i} y_{i}}{4} \approx v_{z}
$$

\section{Trajectory control}

To achieve the path tracking, we suppose that the vehicle position projected in the horizontal plane has already been determined by a localisation procedure $: \mathbf{u}=\left(x_{p}, y_{p}, \theta\right)^{t}$.
The trajectory is specified in the horizontal plane $\left(O, \mathbf{x}_{0}, \mathbf{y}_{0}\right)$ by using a parametric function $\left(x_{d}(t), y_{d}(t)\right)^{t}$. Then, for a given path following velocity, the desired trajectory $\mathbf{u}_{\mathbf{d}}$ can be expressed as a function of time (see Fig. 4):

$$
\mathbf{u}_{\mathbf{d}}=\left(x_{d}, y_{d}, \theta_{d}\right)^{t}
$$

where $\theta_{d}(s)=\arctan \left(\frac{d y}{d t} \frac{d t}{d x}\right)=\arctan \left(\frac{\dot{y}}{\dot{x}}\right)$.

The aim of path tracking control is to compute the vehicle velocity $\mathbf{v}_{\mathbf{t}}=\left(v_{x}, v_{y}, \dot{\theta}\right)^{t}$ such that the path tracking error $\Delta \zeta$ will converge to zero. This path tracking error is defined as the projection on the local vehicle frame of the position error $\boldsymbol{\Delta} \mathbf{u}=\mathbf{u}_{\mathbf{d}}-\mathbf{u}$ :

$$
\boldsymbol{\Delta} \boldsymbol{\zeta}=\left(\begin{array}{ccc}
\mathrm{C}_{\theta} & \mathrm{S}_{\theta} & 0 \\
-\mathrm{S}_{\theta} & \mathrm{C}_{\theta} & 0 \\
0 & 0 & 1
\end{array}\right)\left(\begin{array}{c}
x_{d}-x_{p} \\
y_{d}-y_{p} \\
\theta_{d}-\theta
\end{array}\right)=\mathbf{R}_{\theta} \boldsymbol{\Delta} \mathbf{u}
$$

where $\mathbf{R}_{\boldsymbol{\theta}}$ is the rotation matrix of angle $\theta$ around $\mathbf{z}_{\mathbf{0}}$ axis.

Path tracking control of mobile robots is not a trivial problem[18], [19]. But in our case, since the Hylos platform is omni-directional, we can use a simple proportional strategy to control the vehicle velocity:

$$
\mathbf{v}_{\mathbf{t}}=\mathbf{K}_{\mathbf{t}} \Delta \zeta
$$

where $\mathbf{K}_{\mathbf{t}}=\mathbf{D I A G}\left(K_{x}, K_{y}, K_{\theta}\right)$ is a positive $3 \times 3$ diagonal gain matrix. The control strategy can be adapted through the choice of these gains.

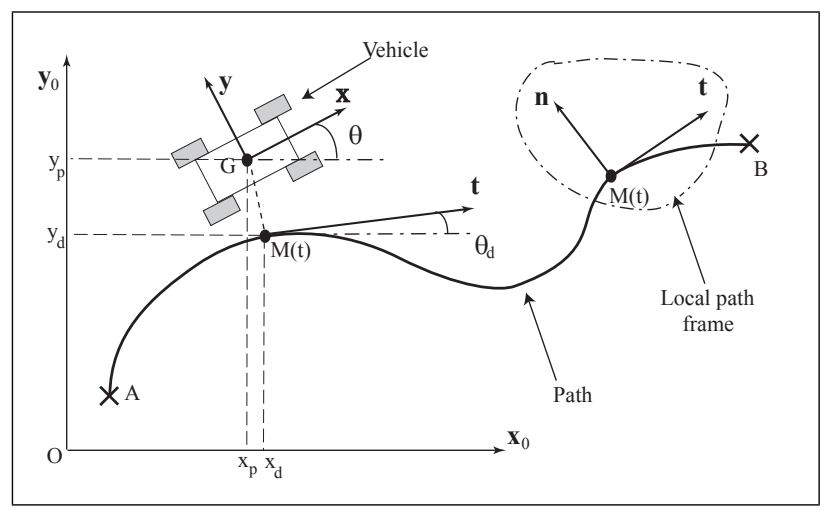

Fig. 4. Path tracking model

\section{Global control strategy}

In the previous section, we introduced the decoupling matrix $\mathbf{D}$ such as $\mathbf{v}_{\mathbf{p}}=\mathbf{D}\left(v_{x}, v_{y}, v_{z}, \dot{\varphi}, \dot{\psi}, \dot{\theta}\right)^{t}$. Considering the velocity correction terms $\left(\mathbf{v}_{\mathbf{t}}, \dot{\mathbf{p}}\right)$, the operational space reconfiguration velocity can be computed as:

$$
\begin{aligned}
& \mathbf{v}_{\mathbf{p}}=\mathbf{D}\left(\mathbf{C}_{\mathbf{p}} \dot{\mathbf{p}}+\mathbf{C}_{\mathbf{t}} \mathbf{v}_{\mathbf{t}}\right) \\
& \dot{x}_{i}=\mathbf{C}_{\mathbf{x}_{\mathbf{i}}} \dot{\mathbf{p}}
\end{aligned}
$$

where $\mathbf{C}_{\mathbf{p}}, \mathbf{C}_{\mathbf{t}}$ and $\mathbf{C}_{\mathbf{x}_{\mathbf{i}}}$ are the corresponding component selection matrices. Then, the joint velocities are computed from this operational velocity vector by considering the inverse velocity model described in Section II. 


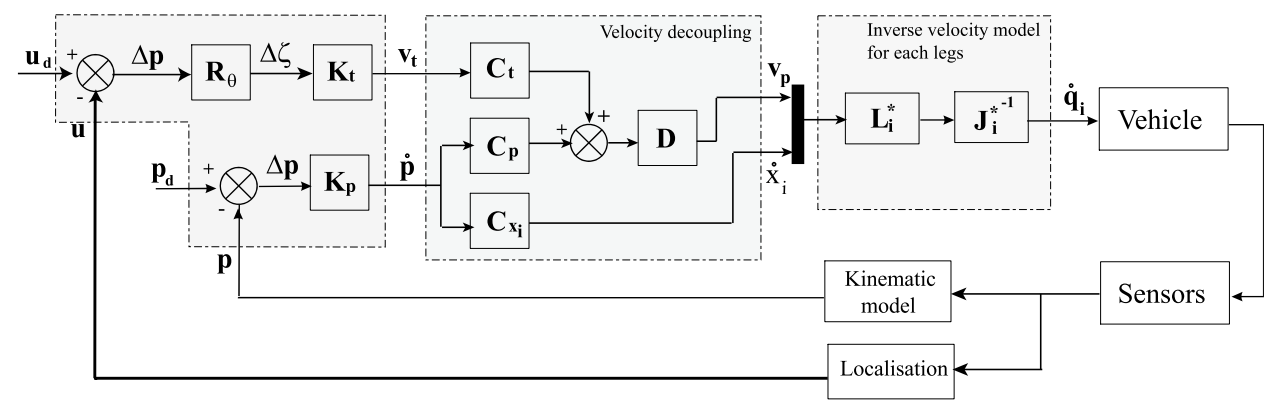

Fig. 5. Posture control scheme

This model is based on the knowledge of contact normal vectors $\mathbf{n}_{\mathbf{i}}$. Equation (9) shows that $\mathbf{t}_{\mathbf{i}}$ can be estimated if the absolute platform velocity $(\mathbf{v}, \boldsymbol{\omega})$ and leg's joint velocities $\left(\dot{\alpha}_{i}, \dot{\beta}_{i}\right)$ are measured. However, this estimation is theoretically independent from the wheel's rotation rate $\omega_{i}$. But for experimental tests, we use a simplified inverse velocity model which is based on a contact normal vector computed from the average plane to contact point $P_{i}$ (see Fig. 2). Finally, the joint velocities are computed using equation (14).

\section{Simulation AND EXPERIMENTAL RESUlTS}

In order to evaluate the control algorithm, both dynamic simulations and experimental tests have been done. The aim of these experimentations is to qualify the practicability of this control method for a given locomotion task which is specified by considering an optimal posture and a given reference path. The presented results concern the correction of the pitch and roll angles of the vehicle Hylos evolving on sloping ground.

The desired posture is specified by considering the optimization of specific locomotion performance criteria. These are the tipover stability margin, and the wheel-ground contact forces balance. The first criterion is of primary importance for the system reliability and the second one improves the global traction performance. We have presented, in previous work, a method to find a suboptimal posture with respect to these criteria[20]: it consists in keeping the platform in the horizontal plane $(\varphi=0, \psi=0)$ and in determining the wheelbase $x_{i}$ in such way that the projected distance, on horizontal plane, between the platform c.o.g and the geometric center of wheel-ground contact points is minimized.

Fig. 6 shows a simulation of Hylos robot evolving on irregular terrain. The results presented in Fig. 7 show that the platform stays horizontally with a quite good accuracy when the robot is moving (the pitch and roll angle error is less then $3^{\circ}$ ).

Experiments have also been conducted with the Hylos robot, which is equipped with a two axis inclinometer measuring the platform pitch and roll angles. In this experiment, the robot is moving at a speed of $0.15 \mathrm{~m} / \mathrm{s}$ on a terrain constituted of slopes with various inclines such that both the pitch and roll angles are changing during the motion. The desired posture is still $\psi=0$ and $\varphi=0$. In Fig. 8 and Fig. 9, the dashed curves represent the vehicle pitch and roll angles when posture

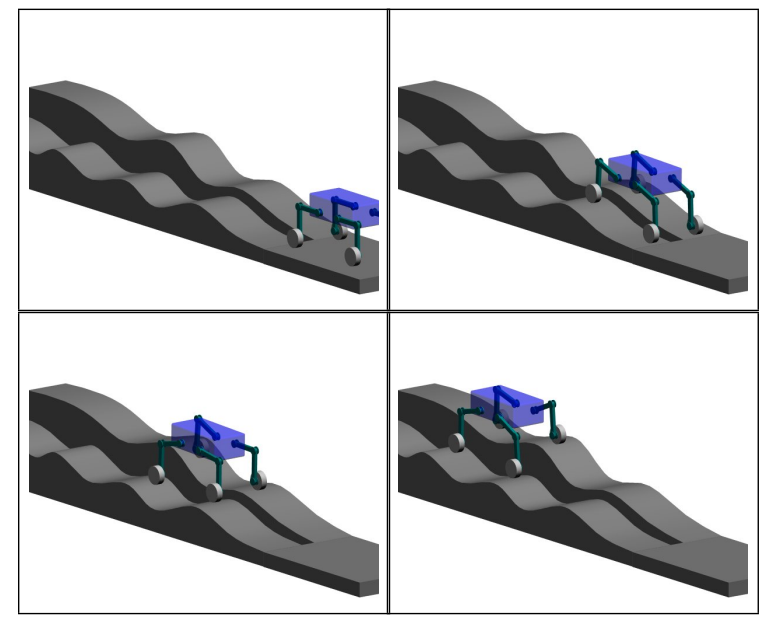

Fig. 6. Simulation illustrations

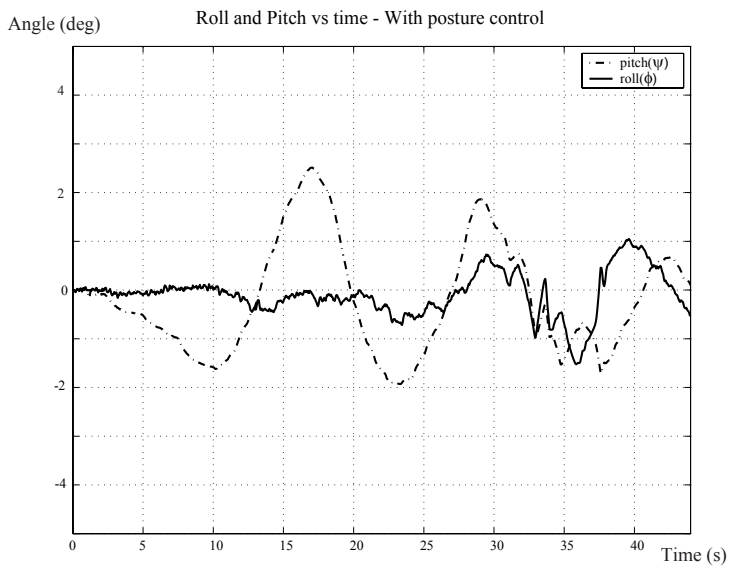

Fig. 7. Simulation results

control is active, and the solid curves is an estimation of the equivalent ground slope angles in pitch an roll directions. The maximum error on corrected angles (the peak on each plot) is partially due to the response time of the feedback control $(10 \mathrm{~Hz})$ and partially due to the velocity limit of the leg's actuators.

\section{CONCLUSION}

In this paper, we have addressed the combined posture and trajectory control of the wheel-legged robot Hylos. An orig- 


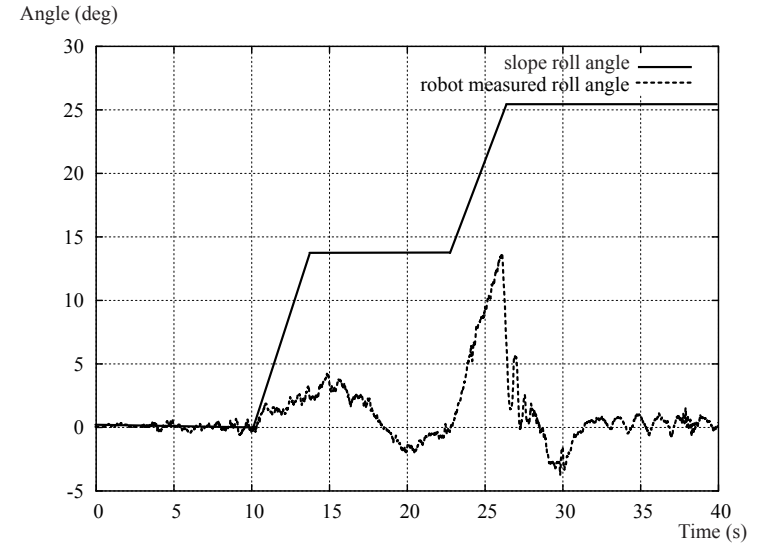

Fig. 8. Robot and ground pitch angles

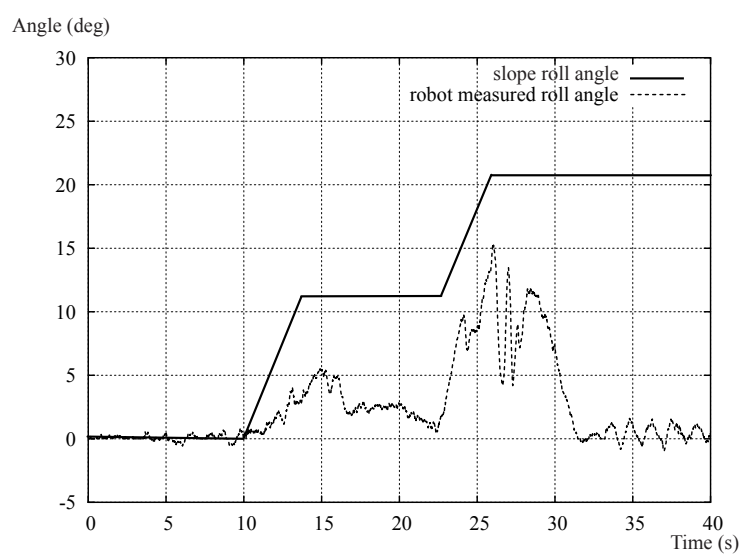

Fig. 9. Robot and ground roll angles

inal velocity based control algorithm has been presented. This method allows to specify the locomotion task in terms of: first the path tracking control and secondly the posture reconfiguration control. A velocity based model has been presented in order to combine the two controls (trajectory and posture) and compute the robot joint velocities. This method is simple to implement as it needs only few sensors that are inclinometers for the pitch-roll measurements and position sensors for the leg mechanisms. The algorithm has been validated through simulations showing the capabilities of such redundantly actuated robot to maintain a certain posture configuration. The practical feasibility of this control algorithm was evaluated and validated through experiments with the

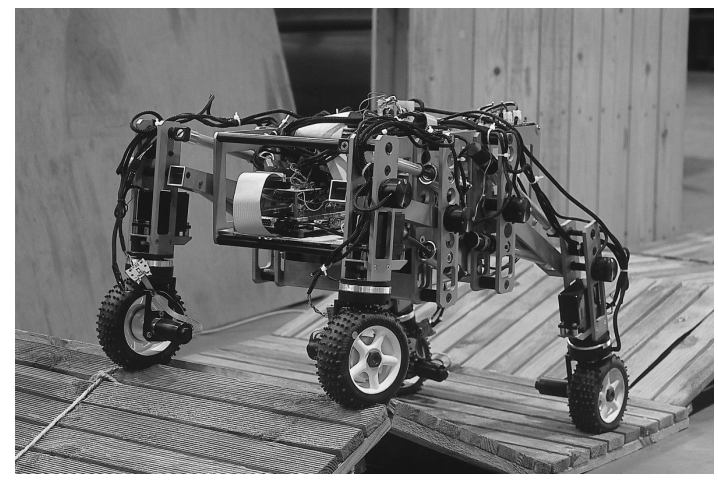

Fig. 10. Experimentations with Hylos
Hylos robot.

This study could be improved by introducing the measurements of forces at each wheel-ground contact. The knowledge of these forces is a possible way to estimate the ground contact angles and then to enhance the posture control. Furthermore, measurement of the normal component should be introduced in a control scheme to insure the permanent contact condition.

\section{REFERENCES}

[1] E. Rollins, J. Luntz, A. Foessel, B. Shamah, and W. Whittaker, "Nomad: a demonstration of the transforming chassis," in IEEE International Conference on Robotics and Automation, 1998, pp. 611-617.

[2] R. Volpe, "Rocky 7: A next generation mars rover prototype," Journal of Advanced Robotics, vol. 11, no. 4, pp. 341-358, 1997.

[3] T. Estier, Y. Crausaz, B. Merminod, M. Lauria, R. Piguet, and R. Siegwart, "An innovative space rover with extended climbing abilities," in International Conference on Robotics in Challenging Environments, Albuquerque, USA, 2000.

[4] R. McGhee, "Finite state control of quadruped locomotion," in Proceedings of the International Symposium on External Control of Human Extremities, 1966.

[5] S. Song and K. Waldron, Machines that walk: the adaptative suspension vehicle. The MIT press, 1989.

[6] S. Sreenivasan and K. Waldron, "Displacement analysis of an actively articulated wheeled vehicule configuration with extensions to motion planning on uneven terrain," Transactions of the ASME, vol. 118, no. 6, pp. 312-317, 1996.

[7] G. Andrade, F. BenAmar, P. Bidaud, and R. Chatila, "Modeling wheelsand interaction for optimization of a rolling-peristaltic motion of a marsokhod robot," in International Conference on Intelligent Robots and Systems, 1998, pp. 576-581.

[8] A. Kemurdjian, "Planet rover as an object of the engineering design work," in IEEE International Conference on Robotics and Automation Belgium, 1998, pp. 140-145.

[9] S. Hirose and H. Takeuchi, "Study on roller-walk (basic characteristics and its control)," in IEEE Int. Conference on Robotics and Automation, 1996, pp. 3265-3270.

[10] A. Halme, I. Leppänen, S. Salmi, and S. Ylönen, "Hybrid locomotion of a wheel-legged machine," in International Conference on Climbing and Walking Robots, Madrid, Spain, 2000.

[11] M. F. and al., "Azimut, a leg-track-wheel robot," in IEEE Int. Conference on Intelligent Robots and Systems, 2003, pp. 2553-2558.

[12] U. Saranli, M. Buehler, and D. Koditschek, "Rhex: a simple and highly mobile hexapod robot," Int. J. Robotics Research, vol. 20, pp. 616-631, 2001.

[13] F. BenAmar, V. Budanov, P. Bidaud, F. Plumet, and G. Andade, "A high mobility redundantly actuated mini-rover for self adaptation to terrain characteristics," in 3rd International Conference on Climbing and Walking Robots, Madrid, Spain, 2000, pp. 105-112.

[14] C. Grand, F. BenAmar, and P. Bidaud, "Kinematic analysis and stability optimisation of a reconfigurable legged-wheeled mini-rover," in SPIE Conference on Unmanned ground-vehicle technology IV, Orlando, USA, 2002, pp. 295-303.

[15] S. Sreenivasan and B. Wilcox, "Stability and traction control of an actively actued micro-rover," Journal of Robotics Systems, vol. 11, no. 6, pp. 487-502, 1994.

[16] K. Iagnemma, A. Rzepniewski, S. Dubowsky, and P. Schenker, "Control of robotic vehicles with actively articulated suspensions in rough terrain," Autonomous Robots, vol. 14, no. 1, pp. 5-16, 2003.

[17] J. Craig, Introduction to Robotics. Addison-Wesley, 1989.

[18] C. G., G. Bastin, and B. D'Andra-Novel, "Structural properties and classification of kinematic and dynamic models of wheeled mobile robots," IEEE Tran. on Robotics and Automation, vol. 12, no. 1, pp. 47-62, 1996.

[19] E. E., H. X., and S. A., "Control of mobile platforms using a virtual vehicle approach," IEEE Tran. on Automatic Control, vol. 46, no. 11, pp. 1777-1782, 2001.

[20] C. Grand, F. BenAmar, F. Plumet, and P. Bidaud, "Stability control of a wheel-legged mini-rover," in 5th International Conference on Climbing on Walking Robots, Paris, France, 2002, pp. 323-331. 